\title{
Avaliação de parâmetros FAD-Cl e seus impactos na vida de fadiga de misturas asfálticas
}

\section{Evaluation of DASR-IC parameters and their influence on the fatigue life of asphalt mixtures}

\author{
Jardel Andrade de Oliveira', Jorge Barbosa Soares ${ }^{2}$, Luis Alberto Herrmann do Nascimento 3 \\ 1Universidade do Ceará, Ceará - Brasil, jardel@det.ufc.br \\ 2Universidade do Ceará, Ceará - Brasil, jsoares@det.ufc.br \\ 3Universidade do Ceará, Ceará - Brasil, luisnascimento@petrobras.com.br
}

\section{Recebido:}

16 de agosto de 2019

Aceito para publicação:

30 de novembro de 2016

Publicado:

2 de dezembro de 2021

Editor de área:

Francisco Sacramento Aragão

\section{Palavras-chave:}

Pavimentos asfálticos.

Misturas.

Fadiga.

Dano.

Modelagem.

Keywords:

Asphaltic pavements.

Mixtures.

Fatigue.

Damage.

Modeling.

DOI:10.14295/transportes.v28i4.2017

\begin{abstract}
RESUMO
Entre as patologias mais comuns em pavimentos estão a deformação permanente e o trincamento por fadiga de misturas asfálticas a quente usadas no revestimento. Os defeitos destacados ainda carecem de metodologias laboratoriais simples e consistentes capazes de apontar misturas com maior potencial de resistência. Há teorias que contribuem, ainda na fase de projeto, para a seleção de granulometrias e de outros parâmetros, com vistas a produzir misturas com maior capacidade de resistir a defeitos em campo. A FAD-Cl (Faixa de Agregados Dominantes, considerando os Componentes Intersticiais) é uma promissora metodologia de seleção granulométrica. O presente trabaIho tem como objetivo avaliar a capacidade de 6 misturas, observando se aquelas que atendem os critérios da metodologia investigada apresentam comportamento superior quanto à fadiga. Para isso, selecionaram-se misturas de um banco de dados composto por 54 misturas, de forma que a primeira mistura não atendesse qualquer dos parâmetros e, paulatinamente, os parâmetros fossem sendo atendidos. Após os ensaios laboratoriais e a avaliação das simulações das vidas de fadiga, concluiu-se que as misturas que atendiam a mais parâmetros da metodologia FAD-Cl apresentaram maior resistência à fadiga.
\end{abstract}

\begin{abstract}
Among the most common distresses in pavements are rutting and fatigue cracking of HMA (Hot Mixture Asphalt) used in surface courses. These defects still lack simple and consistent laboratory methodologies capable of indicating HMA characteristics for higher performance. There are theories that contribute, still in the design phase, to the selection of aggregate gradation and other parameters, to produce HMAs with greater capacity to withstand field distresses. The DASR-IC (Dominant Aggregates Size Range, considering Interstitial Components) is a promising gradation selection methodology. The present work aims to evaluate the capacity of $6 \mathrm{HMAs}$, evaluating whether those that meet the criteria of the investigated methodology have superior performance with respect to fatigue cracking. Mixtures from a database of $54 \mathrm{HMAs}$ were selected, so that the first mixture did not meet any of the mentioned criteria and, gradually, the investigated parameters met the required criteria. After laboratory tests and fatigue life simulations, it was concluded that HMAs that met most of the parameters from the DASR-IC methodology presented greater fatigue resistance.
\end{abstract}

\section{INTRODUCÃO}

Segundo a WHO (2018) (World Health Organization - Organização Mundial da Saúde), os acidentes de trânsito são responsáveis pela morte de mais de um milhão de pessoas por ano. Embora os acidentes possam ser causados por diferentes motivações, a má condição do pavimento 
é apontada como importante fator para a geração de sinistros. É sabido que as rodovias nacionais não oferecem bons níveis de serventia aos usuários (CNT, 2018), observando-se não raro nas mesmas a ocorrência de afundamentos plásticos e de trincamentos, respectivamente, deformação permanente e fadiga. Entre as duas patologias, aponta-se a fadiga como a mais desafiadora, pois não há bem estabelecido uma metodologia ou critérios laboratoriais capazes de se relacionarem bem com o surgimento de trincas e a correspondente evolução em campo.

0 DNIT (Departamento Nacional de Infraestrutura de Transportes) normatizou recentemente o ensaio de Fadiga por Compressão Diametral (DNIT 183/2018), que é o mais empregado no Brasil para caracterizar a fadiga de misturas asfálticas. Contudo, a maioria das misturas aplicadas em campo no país permanece não tendo suas vidas de fadiga verificadas, uma vez que não há neste momento uma exigência legal para tal. Ressalte-se ainda que, de acordo com Monismith (1998) e Babadopulos et al. (2015), amostras submetidas ao ensaio de fadiga por compressão diametral tendem a romper por uma maior influência da deformação permanente (fluência) do que propriamente por fadiga. Já o ensaio Controlled Crosshead Direct Tension-Compression (ensaio Tração Direta - TD ou tração-compressão) (American Association of State Highway and Transportation Officials - AASHTO TP 107, 2018), vem sendo apontado com potencial para caracterizar a fadiga de misturas considerando um número maior de variáveis, como temperatura, frequência de carga, além do valor da deformação.

A vida de fadiga de misturas asfálticas é influenciada pelas características dos seus constituintes (forma e mineralogia dos agregados, curva granulométrica, teor e características do ligante). Normalmente não se relaciona a curva granulométrica com a performance de misturas em campo, mas selecionam-se as granulometrias considerando especificações definidas por percentuais passantes em séries de peneiras e nos tamanhos dos agregados. No Brasil, as faixas granulométricas do DNIT (DNIT 031/2006) ou dos Departamentos Estaduais de Rodovias (DER) representam o estado da prática. Há também experiências específicas como, por exemplo, alguns órgãos rodoviários e empresas concessionárias de rodovias, que possuem suas próprias faixas, adaptadas ou não de faixas existentes. Garantir o enquadramento nestas faixas, sem avaliar a capacidade da mistura resistir a defeitos, não é condição suficiente para produzir misturas com desempenhos satisfatórios.

Existem algumas tecnologias capazes de auxiliar na escolha da curva granulométrica de misturas, como, por exemplo, a metodologia FAD (Faixa de Agregados Dominante) (Kim, 2006), que avalia a granulometria e a volumetria de misturas. Inicialmente, este modelo abordava somente a suscetibilidade das misturas à deformação permanente. Recentemente, expandiram-se as recomendações, criando-se o modelo FAD-CI (CI significando Componentes Intersticiais), que visa identificar aspectos das misturas capazes de diminuir a susceptibilidade das mesmas também ao trincamento por fadiga (Guarin, 2009; Greene et al., 2014; Chum e Kim, 2016). Acredita-se que a adoção desta tecnologia ou similares pode auxiliar a produção de misturas com boa performance em campo. 0 presente trabalho focou em identificar aspectos com a capacidade de incrementar a resistência de misturas à fadiga. Foram selecionadas 6 misturas de um banco de dados de 54 misturas, sendo seus constituintes e parâmetros volumétricos analisados considerando as premissas da metodologia FAD-CI de modo a cobrir um amplo espectro de atendimento aos parâmetros da metodologia. As misturas tiveram suas rigidezes e vidas de fadiga caracterizadas, respectivamente pelos ensaios de módulo dinâmico (AASHTO T 342, 2011) e fadiga TD (AASHTO TP 107, 2018). Por fim, com o intuito de comparar as vidas de fadiga das 
misturas selecionadas, simularam-se os seus desempenhos em campo. Nesta etapa, as deformações suportadas por cada mistura, frente a limites fixos de solicitações (curvas de Wöhler), foram comparadas.

\section{FUNDAMENTAÇÃO TEÓRICA}

\subsection{Fadiga, Dano e Trincamento}

Segundo a ASTM E1823 (2013) (American Society for Testing and Materials), a fadiga pode ser definida como o processo de mudança estrutural permanente, progressivo e localizado, que ocorre em um ponto do material sujeito a tensões de amplitudes variáveis que produzem as fissuras que, por sua vez, se transformam na falha após um determinado número de ciclos. 0 dano por fadiga em misturas asfálticas pode ser compreendido como uma perda progressiva de resistência do material quando submetido a um número de repetições de carga. Vários autores (Kim et al., 2009; Babadopulos, 2014; Nascimento, 2015; Almeida, 2019) vêm estudando fadiga de misturas asfálticas sob a ótica da teoria da Mecânica do Dano Contínuo Viscoelástico (Viscoelastic Continuum Damage - VECD). Esta teoria faz uso de propriedades fundamentais das misturas, sendo uma abordagem funcional na qual se assumem simplificações que não reduzem a sua capacidade de previsões de desempenho.

A VECD baseia-se em variáveis internas de estado que quantificam o dano no material, sendo a evolução deste dano descrita por um modelo fenomenológico desenvolvido a partir de ensaios de laboratório. 0 que diferencia um sólido sem dano do mesmo sólido danificado é apenas um valor menor de rigidez. A variável interna de estado (ou parâmetro de dano) quantifica qualquer alteração microestrutural que resulta em uma redução de rigidez. 0 modelo VECD apoiase em 3 conceitos principais: (i) teoria do trabalho potencial de Schapery, (ii) princípio da correspondência elástica-viscoelástica e (iii) Princípio da Superposição Tempo-Temperatura (PSTT) com dano. Simplificações no cálculo dos parâmetros do modelo VECD e na lei de evolução de dano foram propostas por Underwood et al. (2009), sendo esta abordagem denominada Simplified Viscoelastic Continuum Damage (S-VECD) Model. Uma revisão desses conceitos consta em Nascimento (2015) e em Almeida (2019).

\subsection{Metodologia FAD-CI}

A adoção e a implementação das premissas do modelo FAD-CI são relativamente simples, uma vez que não exigem equipamentos especiais ou a realização de ensaios complexos, baseando-se apenas em informações da granulometria e em propriedades volumétricas da mistura. 0 referido modelo identifica uma faixa de tamanho de agregados dominantes (FAD) como sendo a maior responsável por proporcionar a uma mistura asfáltica uma boa capacidade de resistir à deformação permanente. Os fundamentos são da teoria do empacotamento de partículas (Ferreira, 2015). Os tamanhos de agregados que efetivamente interagem, compondo a FAD, podem ser identificados quando se observa a proporção relativa do percentual de agregados retidos em peneiras consecutivas. Para que um determinado intervalo (duas peneiras consecutivas) de tamanho de agregado pertença a $\mathrm{FAD}$, a referida proporção deve permanecer entre 2,33 $(70 / 30)$ e 0,43 (30/70). Intervalos de tamanhos de agregados que não se enquadrem nas proporções indicadas, tem o espaçamento entre suas partículas aumentado, reduzindo assim os pontos de contato entre as demais partículas da mistura. Dessa forma, os maiores intervalos de tamanhos de partículas, maiores do que a FAD, apenas flutuam dentro da mistura, diminuindo assim os pontos de contato (interação) dos demais tamanhos de agregados (Kim, 2006). 
Os agregados que flutuam na mistura são chamados de flutuantes. Existem ainda os Componentes Intersticiais (CI), que consistem, combinadamente, dos seguintes componentes: agregados menores do que a FAD, Ligante Asfáltico (LA) e Volume de Vazios (Vv). Os CIs mantêm as partículas unidas, atribuindo adesão e resistência à tração (Greene et al., 2014). As propriedades dos CIs influenciam o trincamento por fadiga e a energia de ruptura das misturas (Guarin, 2009; Portela Neto, 2018). Segundo Guarim (2009), para avaliar a estabilidade geral da estrutura da FAD, é importante conhecer o volume de partículas do CI que são maiores do que os vazios da FAD, uma vez que essas são as partículas potencialmente rompedoras da FAD.

A metodologia FAD-CI tem potencial para apontar misturas teoricamente mais resistentes a defeitos (deformação permanente e fadiga). Tal fato se dá pela consideração dos seguintes critérios: (i) Porosidade FAD ( $\eta$ FAD); (ii) Fator de Ruptura (FR); (iii) Razão de Agregados Finos (RAF); e (iv) Espessura Efetiva do Filme de Ligante (EEFL). Os intervalos ótimos são apresentados na seção de resultados. Para a obtenção de maiores detalhes recomenda-se a leitura de Ferreira (2015), Chun e Kim (2016) e Portela Neto (2018). Todas as equações para cálculo dos parâmetros FAD-CI podem ser encontradas em Greene et al. (2014). A Figura 1 apresenta um esquema ilustrativo dos agregados que compõem a FAD (dominantes), os CIs e os agregados flutuantes (partículas maiores do que a FAD).

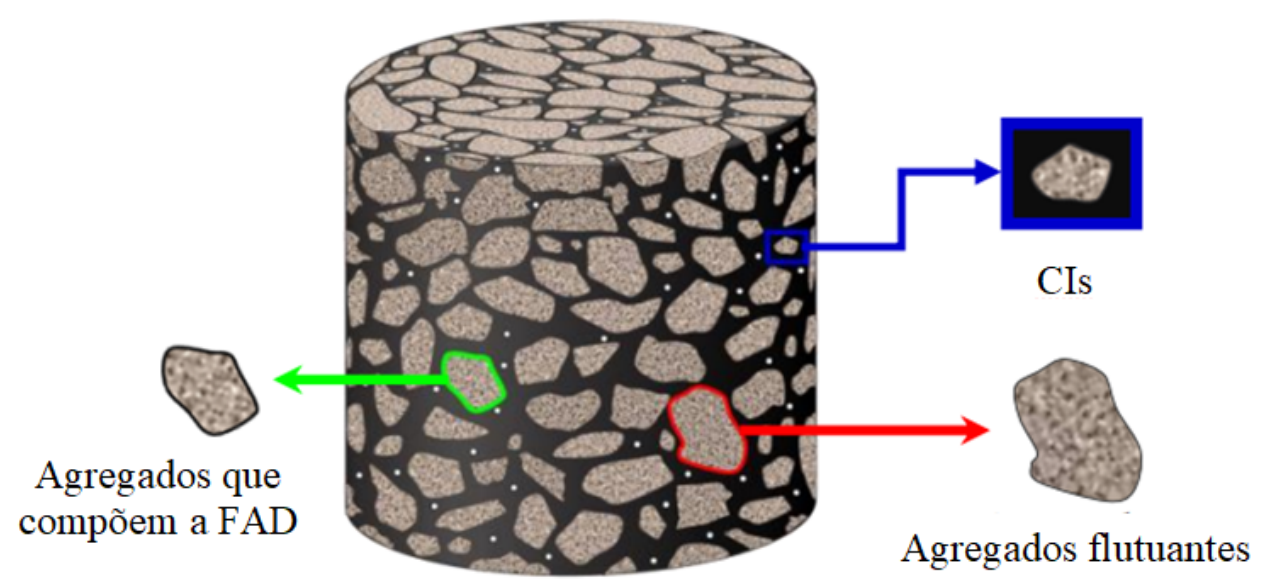

Figura 1. Identificação das partículas na metodologia FAD-Cl (Portela Neto, 2018)

O conceito da espessura do filme de LA surgiu na década de 1940, sendo que vários estudos tentaram, sem sucesso, correlacionar este parâmetro com a durabilidade de misturas (Guarim, 2009). Já o conceito de EEFL foi apresentado por Nukunya et al. (2001). Sengoz e Topal (2007) encontraram uma boa relação entre a espessura do filme de LA e a resistência à tração indireta das misturas envelhecidas. Estudos desenvolvidos por Zhou et al. (2006) mostraram que o Performance Grade (PG) do LA, o teor de LA, a EEFL e a área de superfície dos agregados tiveram impacto significativo na resistência à fadiga de misturas.

No que toca a parâmetros volumétricos comumente usados no Brasil, segundo Fengler (2018), menores valores de RBV (Relação Betume Vazios) acarretam em pequenas espessuras de filme de LA que, por sua vez, aumentam o atrito interno das partículas de misturas e suas rigidezes. De acordo com o referido autor, misturas com baixos valores de RBV apresentam-se quebradiças e com aparente menor durabilidade. 


\section{MATERIAIS E MÉTODOS}

As misturas avaliadas no presente artigo são oriundas de diferentes partes do país, portanto, compostas por ligantes e agregados diferentes. Elas integram o banco de dados da Rede de Tecnologia em Asfaltos (RTA) e foram cedidas pela Petrobras. De um banco de dados composto por 54 misturas, foram selecionadas 6 (M1, M2, M3, M4, M5 e M6) de acordo com seus enquadramentos nos parâmetros da metodologia FAD-CI. Foram avaliados os parâmetros FAD-CI de todo o banco de dados de misturas para que fossem escolhidas as 6 aqui investigadas. Inicialmente, para a M1, buscou-se uma mistura que não atendesse quaisquer dos critérios relativos aos parâmetros mencionados. Já para a M2, buscou-se uma que atendesse apenas a porosidade, $\eta$ FAD. Para a M3, buscou-se uma mistura que atendesse a $\eta$ FAD e o FR. Seguindo essa lógica, onde cada mistura deveria atender um parâmetro adicional, a M5 deveria atender todos os parâmetros ( $\eta$ FAD, FR, RAF, EEFL). Contudo, as misturas do banco de dados que atendiam o critério para EEFL, atendiam apenas este parâmetro. Dessa forma, a M5 atende apenas o EEFL. Além de M5, apenas uma outra mistura do banco de dados atendia o critério EEFL e, da mesma forma que M5, esta satisfazia apenas este critério. Logo, incluiu-se a referida mistura nas análises (M6) com o intuito de melhor avaliar a capacidade do parâmetro EEFL relacionar-se com o dano por fadiga.

A origem mineralógica dos agregados, Tamanho Máximo Nominal (TMN) e alguns parâmetros volumétricos constam na Tabela 1, que apresenta ainda os critérios da metodologia FAD-CI. Células assinaladas com " $\mathrm{x}$ " e com "Ok" significam critério não atendido e critério atendido, respectivamente.

Tabela 1 - Características das misturas e critérios FAD-Cl

\begin{tabular}{|c|c|c|c|c|c|c|c|c|c|c|c|}
\hline \multirow{2}{*}{ Misturas } & \multicolumn{2}{|c|}{ CAP* } & \multirow{2}{*}{$\begin{array}{l}\mathrm{Vv} \\
(\%) \\
\end{array}$} & \multirow{2}{*}{$\mathrm{G}_{\mathrm{mm}}$} & \multirow{2}{*}{ Origem } & \multirow{2}{*}{$\begin{array}{l}\text { Fíler } \\
\text { (Cal) }\end{array}$} & \multirow{2}{*}{$\begin{array}{l}\text { TMN } \\
(\mathrm{mm}) \\
\end{array}$} & \multicolumn{4}{|c|}{ Critérios FAD-Cl } \\
\hline & Pen. & Teor (\%) & & & & & & $\eta$ FAD & $\mathrm{FR}$ & RAF & EEFL \\
\hline M1 & 57 & 5,5 & 4,2 & 2,532 & Sienito & & 12,7 & $x$ & $x$ & $x$ & $x$ \\
\hline $\mathrm{M} 2$ & 43 & 5,5 & 4,0 & 2,631 & Granítico & & 12,7 & Ok & $x$ & $x$ & $x$ \\
\hline M3 & 61 & 4,7 & 4,0 & 2,700 & - & - & 12,7 & Ok & Ok & $x$ & $x$ \\
\hline M4 & 50 & 4,9 & 3,8 & 2,591 & Granítico & & 12,7 & Ok & Ok & Ok & $x$ \\
\hline M5 & 50 & 4,1 & 5,0 & 3,027 & Basáltico & $1,5 \%$ & 19,0 & $x$ & $x$ & $x$ & Ok \\
\hline M6 & 46 & 5,0 & 5,0 & 2,621 & Calcária & $2,0 \%$ & 25,0 & $x$ & $x$ & $x$ & Ok \\
\hline
\end{tabular}

Para avaliar a relevância na vida de fadiga dos parâmetros FAD-CI de forma isolada, desejavase selecionar misturas compostas por agregados de mesma origem, com os mesmos TMNs e tipo de CAP, e que diferissem apenas pelo enquadramento nos critérios FAD-CI. Porém, não foi possível identificar a origem mineralógica dos agregados de M3. Já M5 e M6 tem TMN maiores que as demais misturas, além de serem as únicas misturas a conter fíler artificial (1,5\% e 2,0\% de cal, respectivamente) em suas composições, características que afetam a resistência à fadiga. A influência que cada critério da metodologia FAD-CI exerce sobre a resistência da mistura ainda é objeto de pesquisa, como a reportada neste artigo.

Pela realização do ensaio de Modulo Dinâmico e pela consideração do PSTT (Princípio da Superposição Tempo-Temperatura), foram obtidas as curvas mestras das misturas. Nesta etapa optou-se pelo uso da equação de Williams-Landel-Ferry (WLF) (Equação 1).

$$
\log \left(a_{T}\right)=\frac{-C_{1} \cdot\left(T-T_{R E F}\right)}{C_{2}+\left(T-T_{R E F}\right)}
$$


Onde: $a_{T}=$ time-temperature shift fator; $C_{1}$ e $C_{2}=$ constantes de ajuste da curva; $T=$ temperaturas de obtenção dos dados; $T_{R E F}=$ temperatura de referência para construção da curva. Para a obtenção das curvas mestras realizaram-se ensaios de módulo dinâmico com três valores (4, 20 e $40^{\circ} \mathrm{C}$ ) de temperatura $(T)$. Utilizou-se $20^{\circ} \mathrm{C}$ como temperatura de referência $\left(T_{R E F}\right)$.

As vidas de fadiga das misturas foram avaliadas pelo ensaio TD com o modelo S-VECD. Utilizou-se critério de falha baseado na taxa média de pseudoenergia liberada por ciclo $\left(G^{R}\right)$. Foram empregadas as Equações 2 e 3 para gerar as curvas características dos danos ( $\mathrm{C}$ vs $\mathrm{S}$ ) e as curvas $G^{R} v s N_{f}$ de cada mistura. Para obter mais detalhes sobre o conceito das curvas C vs S e $G^{R} v s N_{f}$, recomenda-se a leitura de Nascimento (2016).

$$
\begin{gathered}
C(S)=1-C_{11} S^{C_{12}} \\
G^{R}=Y N_{f}{ }^{\Delta}
\end{gathered}
$$

Onde: $\mathrm{C}_{11}$ e $\mathrm{C}_{12}=$ coeficientes da curva $C$ v $S ; G^{R}=$ taxa de variação média da energia de pseudo-deformação liberada (por ciclo) durante a execução do ensaio; $Y$ e $\Delta=$ coeficientes de ajuste do critério de ruptura $G^{R} v s N_{f} ; N_{f}=$ número de ciclos até a falha.

Com o intuito de avaliar a capacidade de cada mistura resistir à fadiga foram utilizadas as equações descritas em Nascimento (2016), que simulam a previsão da vida de fadiga $\left(N_{f}\right)$ e do parâmetro de dano, a partir de dados do ensaio de fadiga TD [Equações (2) a (7)].

$$
\begin{gathered}
N_{f}=\left(\frac{\delta}{Y\left(\frac{C_{12}}{p}+1\right)}\right)^{\frac{1}{\Delta+1-\frac{C_{12}}{p}}} \\
\delta=\frac{1}{2}\left(\varepsilon_{T A}^{R}\right)^{2} C_{11}\left(\frac{p\left(C_{11} C_{12}\right)^{\alpha}\left(\varepsilon_{T A}^{R}\right)^{2 \alpha}}{f_{R^{2}}{ }^{\alpha}}\right)^{\frac{C_{12}}{p}} N^{\frac{C_{12}}{p}} \\
\varepsilon_{T A}^{R}=\frac{1}{E_{R}} \frac{\beta+1}{2}\left[\left(\varepsilon_{0, p p}\right)_{i}\left|E^{*}\right|_{L V E}\right] \\
p=\alpha-\alpha C_{12}+1
\end{gathered}
$$

Onde: $\mathrm{C}_{11}$ e $\mathrm{C}_{12}=$ coeficientes da curva $C$ vs $S ; Y$ e $\Delta=$ coeficientes de ajuste do critério de ruptura $G^{R}$ v $S N_{f} ; N_{f}=$ número de ciclos até a falha; $\varepsilon_{T A}^{R}=$ amplitude da pseudo-deformação; $\alpha=$ taxa de evolução do dano; $f \mathrm{R}=$ frequência reduzida; $\varepsilon_{0, p} p=$ amplitude de deformação pico a pico; $\beta=$ parcela do ciclo em que ocorre tensão de tração; $\left|E^{*}\right|_{L V E}=$ módulo dinâmico viscoelástico linear nas condições de temperatura e frequência.

A taxa de evolução de dano $\alpha$ é obtida do espectro de relaxação $(\alpha=1+1 / \mathrm{m}$, onde $\mathrm{m}$ indica o máximo valor da derivada do módulo de relaxação em relação ao tempo, em escala log-log). As simulações utilizaram como critério de qualidade as respostas das amplitudes das deformações suportadas por cada mistura frente a valores fixos de $N_{f}$ (parâmetros das Curvas de Wöhler). Com o valor de $N_{f}$ mantido constante, a mistura que apresentar o maior valor de amplitude de deformação pode ser apontada como tendo um melhor comportamento à fadiga. De modo a simplificar a comparação entre as misturas optou-se por valores fixos de $N_{f}\left(1 \times 10^{6}\right.$ e $\left.1 \times 10^{4}\right)$. As simulações foram realizadas considerando as frequências de $25 \mathrm{~Hz}, 10 \mathrm{~Hz}$ e $0,5 \mathrm{~Hz}$ e dois valores de temperatura para representar locais com climas frios e quentes $\left(10^{\circ} \mathrm{C} \mathrm{e} 40^{\circ} \mathrm{C}\right)$. Com o intuito de observar a influência de diferentes valores de temperatura gerou-se uma curva de Wöhler da mistura M4, mantendo-se fixos os valores de frequência em $10 \mathrm{~Hz}$ e variando-se os valores de temperatura. Fez-se uso de metodologia similar para evidenciar a influência da frequência, gerando-se outra curva de Wöhler da mesma mistura, porém mantendo-se constantes os valores de temperatura $\left(10^{\circ} \mathrm{C}\right)$ e variando-se os valores de frequência. Curvas de Wöhler, ou simplesmente curvas de fadiga, relacionam amplitude de deformação com número de ciclos de carregamento. Ainda com o intuito de avaliar a resistência à fadiga empregou-se o Fator de 
Fadiga das Misturas (FFM), parâmetro proposto por Nascimento (2015). 0 referido parâmetro é numericamente igual à área abaixo da Curva de Wöhler (para $20^{\circ} \mathrm{C}$ e $10 \mathrm{~Hz}$ ). A referida área é calculada entre os valores de 100 até 200 microstrains, pois estes são valores de deformação tipicamente encontrados em pavimentos brasileiros [Equação (8)]. Valores maiores de FFM indicam misturas com maiores resistências à fadiga.

$$
F F M=\frac{\left[\log \left(N_{f, 100 \mu \varepsilon}\right)+\log \left(N_{f, 200 \mu \varepsilon}\right)\right]}{2} \times[\log (200)-\log (100)]
$$

Fez-se uso ainda de metodologia proposta por Nascimento (2018), que classifica misturas em 4 diferentes níveis de resistência à fadiga. As classes variam de 1 a 4 , respectivamente, representando as misturas com piores resistências até as misturas com maiores resistências. A referida técnica consiste em relacionar o FFM com a rigidez $\left(\left|E^{*}\right| @ 20^{\circ} \mathrm{C}, 10 \mathrm{~Hz}\right)$ de cada mistura. A Figura 2 apresenta o fluxo das ações para o desenvolvimento deste artigo.

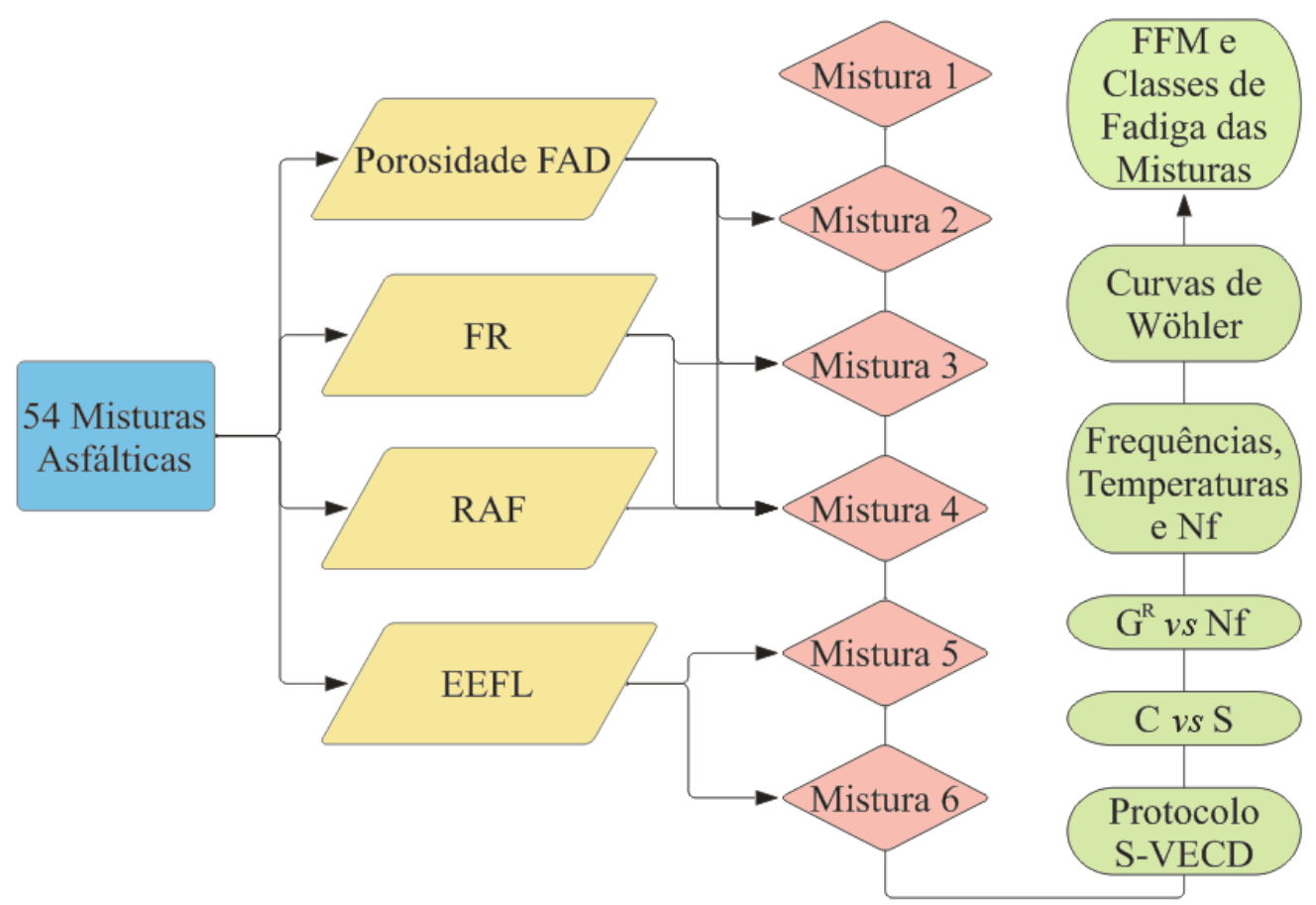

Figura 2. Fluxograma das atividades desenvolvidas

\section{RESULTADOS E DISCUSSÕES}

Os valores obtidos e os intervalos ideais dos parâmetros da metodologia FAD-CI constam na Tabela 2, enquanto a Figura 3a apresenta as curvas características de dano (C vs S) e a Figura $3 \mathrm{~b}$ apresenta as curvas $G^{R} v s N_{f}$ das misturas avaliadas.

Tabela 2 - Critérios para FAD-Cl das misturas avaliadas

\begin{tabular}{|c|c|c|c|c|c|c|c|c|}
\hline \multirow{2}{*}{ Misturas } & \multicolumn{2}{|c|}{$\eta$ FAD (\%) } & \multicolumn{2}{|r|}{ FR } & \multicolumn{2}{|r|}{ RAF } & \multicolumn{2}{|c|}{ EEFL (microns) } \\
\hline & Obt. & Ideal & Obt. & Ideal & Obt. & Ideal & Obt. & Ideal \\
\hline M1 & 64 & & 1,36 & & 0,57 & & 55,8 & \\
\hline M2 & $43^{*}$ & & 0,39 & & 0,26 & & 67,3 & \\
\hline M3 & $43^{*}$ & & $0,83^{*}$ & & 0,47 & & 36,5 & \\
\hline M4 & $44^{*}$ & $38-48$ & $0,81^{*}$ & $0,50-0,95$ & $0,35^{*}$ & $0,28-0,36$ & 37,0 & $2,5-25,0$ \\
\hline M5 & 51 & & 1,20 & & 0,70 & & $24,7^{*}$ & \\
\hline M6 & 61 & & 1,40 & & 0,26 & & $23,7^{*}$ & \\
\hline
\end{tabular}




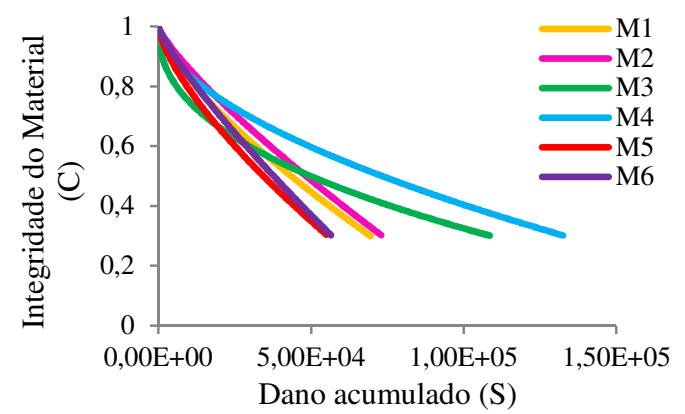

(a) Ajuste das curvas $C$ vs $S$

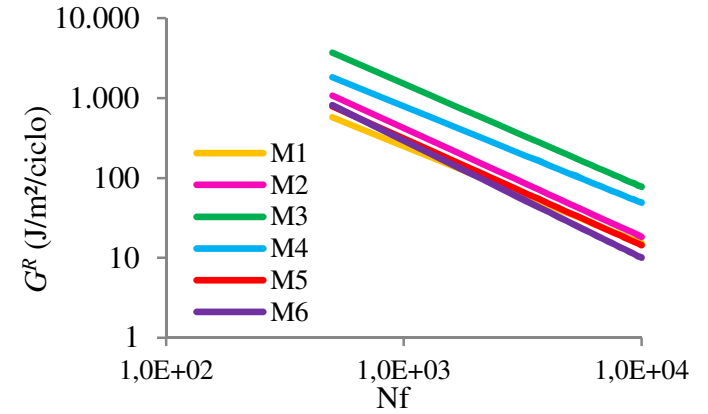

(b) Curvas $G^{R}$ vs $N_{f}$

Figura 3. Curvas características dos danos e curvas $G^{R}$ vs $N_{f}$

Para valores menores do que aproximadamente 0,5 de integridade do material (C), percebese que as curvas das misturas M1, M2, M3 e M4 (Figura 3a), para um mesmo valor de dano acumulado (S), apresentam maiores valores de C. Tal observação corrobora o enquadramento paulatino dos parâmetros FAD-CI das misturas citadas. Já as misturas M5 e M6 apresentam curvas com formatos similares. Estas não seguiram a lógica de enquadramento nos parâmetros FAD-CI das demais (atenderam apenas o parâmetro EEFL), e também não seguiram a tendência observada em M1, M2, M3 e M4, ficando posicionadas abaixo das curvas das demais misturas (Figura 3a). Contudo, é importante destacar que os diferentes valores de rigidez e as diferentes formas de evolução do dano nas misturas $(\alpha)$ não permitem concluir que misturas com maior integridade na curva $\mathrm{C}$ vs $\mathrm{S}$ tenham maior resistência à fadiga. Diferentes inclinações nas curvas GR vs Nf indicam que o dano nas misturas evoluem de forma diferente. Portanto, não é possível utilizar apenas as curvas da Figura 3 para concluir sobre quais misturas apresentam maior resistência à fadiga. A Tabela 3 apresenta os parâmetros da equação WLF, das equações do modelo S-VECD e a taxa de evolução do dano das misturas. Já a Figura 4 apresenta os valores das simulações das vidas de fadiga (parâmetros das curvas de Wöhler). As Figuras 4a e b expõem os valores de amplitude de deformação calculados à $10^{\circ} \mathrm{C}$ e com dois valores de $\mathrm{Nf}(1 \times 106 \mathrm{e}$ $1 \times 104)$ ). Já as Figuras 4c e 4d expõem a mesma propriedade, calculada com os mesmos valores de $\mathrm{Nf}$, porém à $40^{\circ} \mathrm{C}$.

Observando os valores das amplitudes de deformação de M1 e M2 (Figura 4), percebe-se que estes sempre são menores do que os valores das misturas que atenderam mais parâmetros FADCI (M3 e M4), indicando que as referidas misturas têm as menores resistências à fadiga. Nas Figuras 4c e 4d percebe-se uma exceção a esta tendência, pois M2, na frequência de 0,5Hz, mostrou-se superiores a M4. Lembrando que M2 atende apenas a $\eta$ FAD, sendo que este parâmetro supostamente não se relaciona com fadiga e sim com deformação permanente (KIM, 2006). Logo, considera-se que os resultados das simulações de vida de fadiga de M1 e M2 corroboram as indicações da metodologia FAD-CI.

Por sua vez, M3 e M4, as misturas que mais atenderam os critérios FAD-CI, posicionam-se como as mais resistentes à fadiga à $10^{\circ} \mathrm{C}$ nos dois valores de $N_{f}$. Já quando se observa as análises à $40^{\circ} \mathrm{C}$, nota-se que a resistência à fadiga de $\mathrm{M} 4$ apresenta pior performance, sendo superada pelas outras misturas com o menor valor de $N_{f}$ (Figura $4 \mathrm{~d}$ ). Comparando-se M3 e M4 nas 4 configurações de temperatura e $N_{f}$ avaliadas na Figura 4, nota-se que M3 não apresentou desempenho superior a M4 apenas na Figura $4 \mathrm{a}\left(10^{\circ} \mathrm{C}, N_{f}=1 \times 106\right)$. 
Tabela 3 - Parâmetros da equação WLF e das equações do modelo S-VECD

\begin{tabular}{lccccccc}
\hline Misturas & $\mathrm{C}_{1}$ & $\mathrm{C}_{2}$ & $\mathrm{C}_{11}$ & $\mathrm{C}_{12}$ & $Y$ & $\Delta$ & $\alpha$ \\
\hline $\mathrm{M} 1$ & $1,24 \mathrm{E}+01$ & $9,81 \mathrm{E}+01$ & $2,33 \mathrm{E}-04$ & $7,18 \mathrm{E}-01$ & $1,09 \mathrm{E}+06$ & $-1,214$ & 2,94 \\
M2 & $2,49 \mathrm{E}+01$ & $1,94 \mathrm{E}+02$ & $8,27 \mathrm{E}-05$ & $8,07 \mathrm{E}-01$ & $5,06 \mathrm{E}+06$ & $-1,360$ & 2,89 \\
M3 & $2,25 \mathrm{E}+01$ & $1,56 \mathrm{E}+02$ & $4,74 \mathrm{E}-03$ & $4,31 \mathrm{E}-01$ & $1,15 \mathrm{E}+07$ & $-1,293$ & 2,60 \\
M4 & $2,20 \mathrm{E}+01$ & $1,64 \mathrm{E}+02$ & $9,04 \mathrm{E}-04$ & $5,64 \mathrm{E}-01$ & $3,40 \mathrm{E}+06$ & $-1,211$ & 3,50 \\
M5 & $2,10 \mathrm{E}+01$ & $1,48 \mathrm{E}+02$ & $3,19 \mathrm{E}-04$ & $7,04 \mathrm{E}-01$ & $3,16 \mathrm{E}+06$ & $-1,335$ & 2,88 \\
M6 & $2,12 \mathrm{E}+01$ & $1,55 \mathrm{E}+02$ & $8,31 \mathrm{E}-05$ & $8,26 \mathrm{E}-01$ & $7,57 \mathrm{E}+06$ & $-1,470$ & 3,07 \\
\hline
\end{tabular}

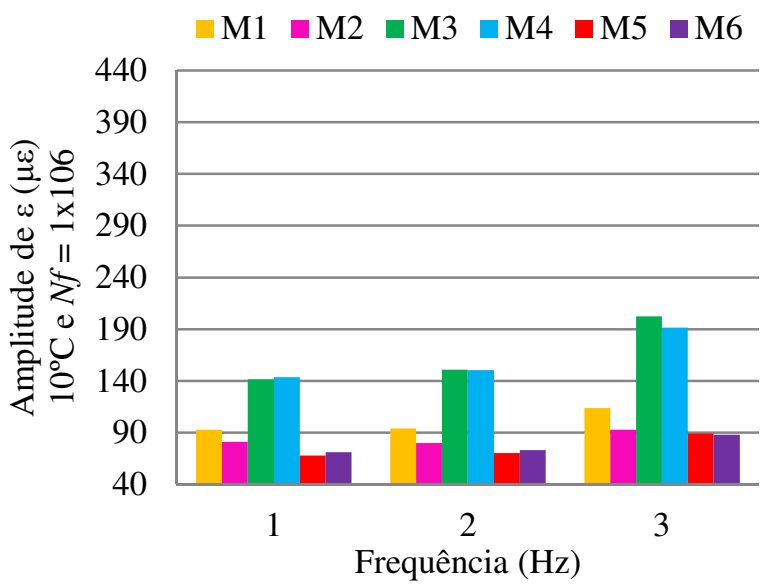

(a) Amplitude de deformação $\left(10^{\circ} \mathrm{C}, N_{f}=1 \times 10^{6}\right)$

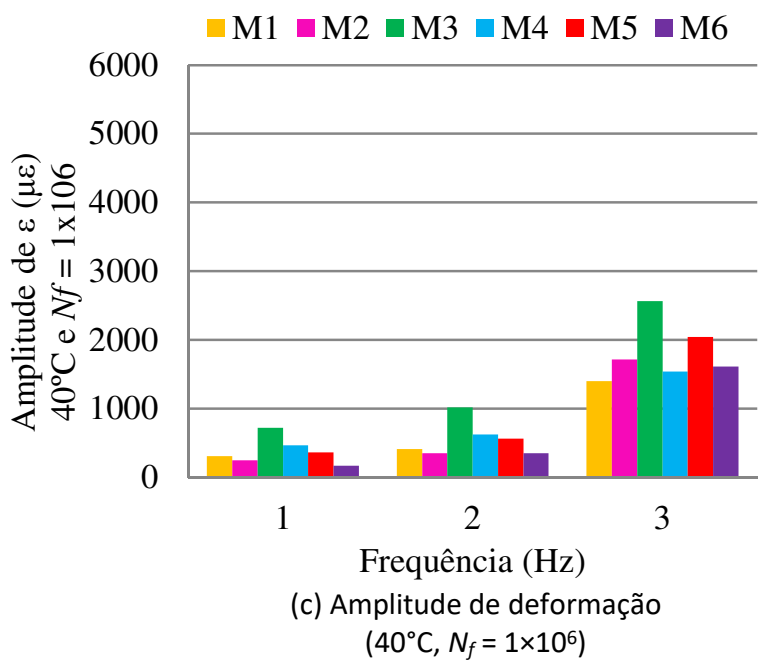

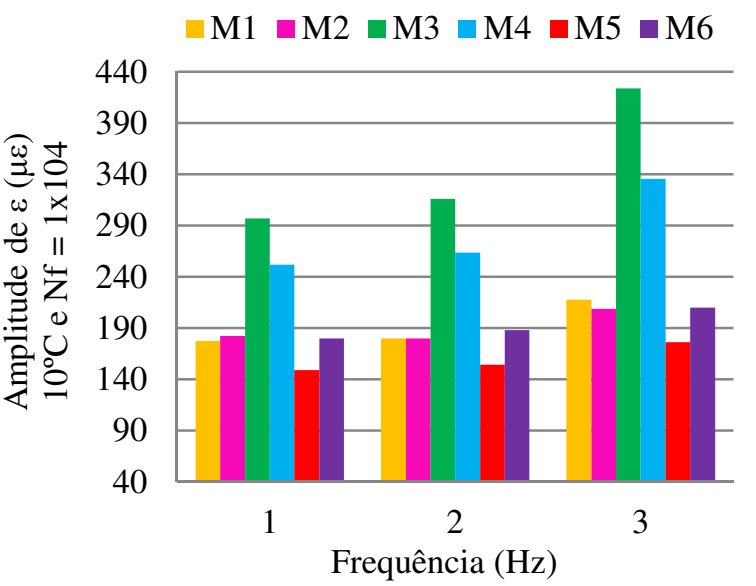

(b) Amplitude de deformação $\left(10^{\circ} \mathrm{C}, N_{f}=1 \times 10^{4}\right)$

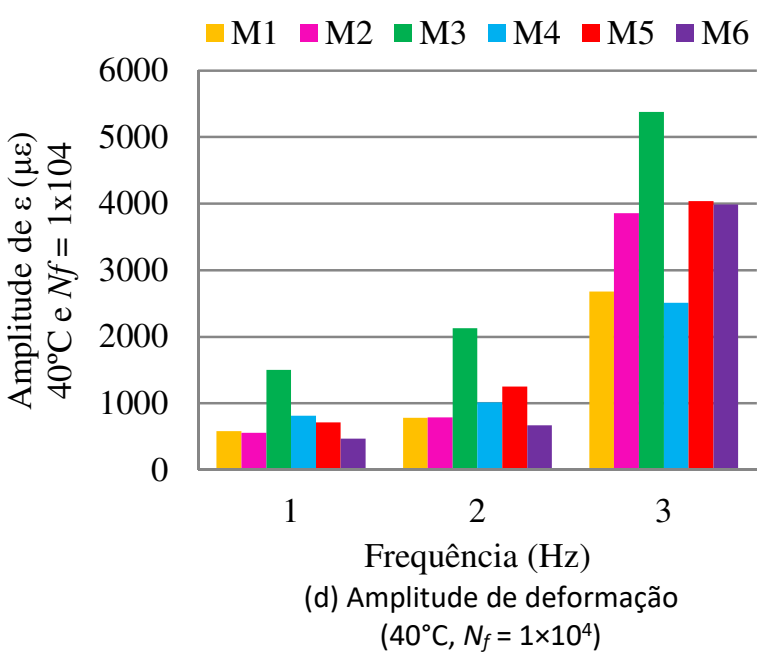

Figura 4. Simulações das vidas de fadiga separadas por temperatura e por $N_{f}$

Observando os valores das amplitudes de deformação de M1 e M2 (Figura 4), percebe-se que estes sempre são menores do que os valores das misturas que atenderam mais parâmetros FADCI (M3 e M4), indicando que as referidas misturas têm as menores resistências à fadiga. Nas Figuras 4c e 4d percebe-se uma exceção a esta tendência, pois $M 2$, na frequência de $0,5 \mathrm{~Hz}$, mostrou-se superiores a M4. Lembrando que M2 atende apenas a $\eta$ FAD, sendo que este parâmetro supostamente não se relaciona com fadiga e sim com deformação permanente (KIM, 2006). Logo, considera-se que os resultados das simulações de vida de fadiga de M1 e M2 corroboram as indicações da metodologia FAD-CI.

Por sua vez, M3 e M4, as misturas que mais atenderam os critérios FAD-CI, posicionam-se como as mais resistentes à fadiga à $10^{\circ} \mathrm{C}$ nos dois valores de $\mathrm{Nf}$. Já quando se observa as análises à $40^{\circ} \mathrm{C}$, nota-se que a resistência à fadiga de M4 apresenta pior performance, sendo superada 
pelas outras misturas com o menor valor de Nf (Figura 4d). Comparando-se M3 e M4 nas 4 configurações de temperatura e Nf avaliadas na Figura 4, nota-se que M3 não apresentou desempenho superior a $\mathrm{M} 4$ apenas na Figura $4 \mathrm{a}\left(10^{\circ} \mathrm{C}, \mathrm{Nf}=1 \times 106\right)$.

Ainda observando a Figura 4, percebe-se que M5 pode ser apontada como a mistura com menor capacidade de resistir à fadiga nas análises a $10^{\circ} \mathrm{C}$, independentemente do valor de $\mathrm{Nf}$ e da frequência. A mesma afirmação não pode ser feita para $\mathrm{M} 6$, pois na Figura $4 \mathrm{~b}\left(10^{\circ} \mathrm{C}, \mathrm{Nf}=\right.$ $1 \times 104$ ), esta mistura apresentou desempenho, embora similar, superior às misturas M1 e M2. É importante destacar que M5 e M6 têm os maiores TMNs, respectivamente $19 \mathrm{~mm}$ e $25 \mathrm{~mm}$, sendo que todas as demais misturas têm os mesmos TMNs (12,7mm). Hou et al. (2010), fazendo uso da metodologia VECD, avaliaram e ranquearam 11 misturas asfálticas. As misturas avaliadas por estes autores com menores TMNs apresentaram-se mais resistentes à fadiga.

Ainda sobre M5 e M6, quando se observa as análises a $40^{\circ} \mathrm{C}$, percebe-se que os seus desempenhos à fadiga são incrementados em alguns momentos (Figuras 4c e 4d), sobretudo nas análises com menores frequências, posicionando-se como as melhores misturas entre as testadas. É importante perceber que, embora o parâmetro EEFL de M5 e M6 esteja enquadrado no intervalo desejável, estas misturas apresentam as menores EEFL. Dessa forma, acredita-se que seus esqueletos pétreos sejam os menos lubrificados e que seus agregados tenham mais pontos de contatos, elevando assim as suas rigidezes e diminuindo as suas resisitências à fadiga. Tal comportamento deve ser incrementado quando submetido a menores valores de temperatura, devido a maior rigidez do LA nessa condição. Já quando as misturas são submetidas a maiores valores de temperatura, seus CAPs tendem a apresentar-se menos viscosos, diminuindo as rigidezes das misturas e incrementando suas resistências à fadiga.

Com o intuito de investigar o impacto da variação da temperatura e da frequência, construíram-se as Curvas de Wöhler (Figura 5). Utilizou-se apenas M4 nesta etapa do trabalho, contudo, com os dados e as equações, as demais curvas de Wöhler podem ser facilmente construídas. Percebe-se que o procedimento adotado se mostrou mais sensível as variações de temperatura do que de frequência. Ao submeter M4 a um valor maior de temperatura e menor de frequência, a mistura resistiu a níveis mais elevados de amplitude de deformação, ou seja, apresentou maior resistência à fadiga. Tal fato pode indicar que, misturas aplicadas em locais com maiores médias de temperatura ou menores frequências de carregamento do tráfego de veículos, tendem a ser mais resistentes à fadiga. É sabido que temperaturas mais elevadas proporcionam menor viscosidade dos ligantes asfálticos e, por conseguinte, menores valores de rigidez das misturas. Dessa forma, acredita-se que as referidas condições podem incrementar a resistência ao trincamento de misturas. Considera-se bastante importante que tal fato seja reconhecido no dimensionamento de pavimentos asfálticos (SANTIAGO et al., 2018, 2019).

A Figura 6a apresenta os valores dos FFM, enquanto a Figura $6 \mathrm{~b}$ apresenta o posicionamento das misturas nas 4 classes de resistência à fadiga propostas por Nascimento (2018). As curvas observadas na Figura 6b separam as classes de fadiga das misturas.

Da Figura 6a, percebe-se que M3 e M4 apresentaram os maiores valores de FFM, logo esperase que sejam as misturas mais resistentes à fadiga. Este fato corrobora com o enquadramento das misturas nos parâmetros da metodologia FAD-CI, uma vez que as referidas misturas atenderam a mais parâmetros. Chama-se atenção para o parâmetro FR (atendido por M3 e M4), principal parâmetro da metodologia FAD-CI para indicar misturas resistentes à fadiga. Já M1, M2, M5 e M6 que se enquadram no intervalo ótimo de apenas um parâmetro ou em nenhum 
(caso de M1), apresentaram valores de FFM mais baixos, indicando menores resistências à fadiga. As referidas quatro misturas não atendem o critério FR, principal parâmetro da metodologia FAD-CI para indicar resistência à fadiga. Já quando se observam as classes das misturas (Figura 6b), percebe-se a mesma tendência observada na Figura 6a, com M3 e M4 tendo maior resistência à fadiga, enquadrando-se na classe 4 . As demais misturas enquadram-se nas classes 1 e 2, que identificam as misturas mais susceptíveis a este defeito, com exceção de M5 que se enquadrou na classe 3, porém muito próxima a classe 2. É importante notar que M4 é a mistura que atende a mais critérios da metodologia FAD-CI, sendo também a mistura melhor posicionada dentro das classes de resistência das misturas.

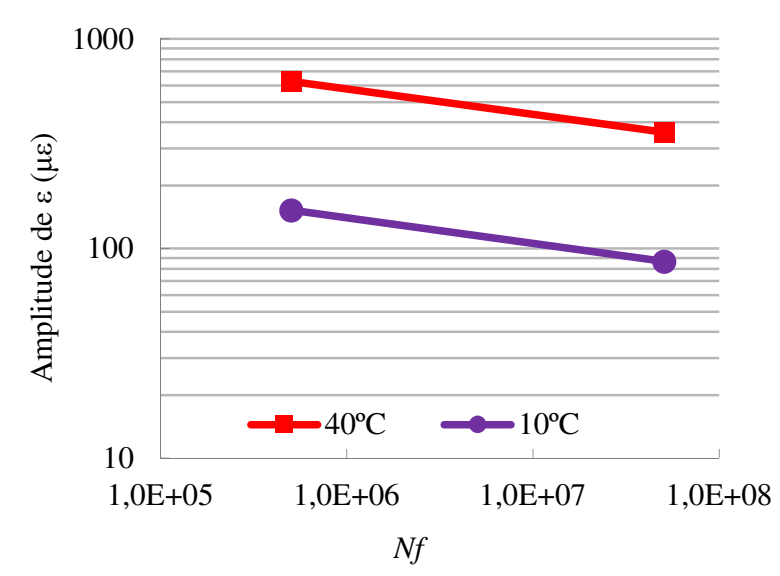

(a) Variação de temperaturas (40 e $10 \circ \mathrm{C})$ e frequência fixa $(10 \mathrm{~Hz})$

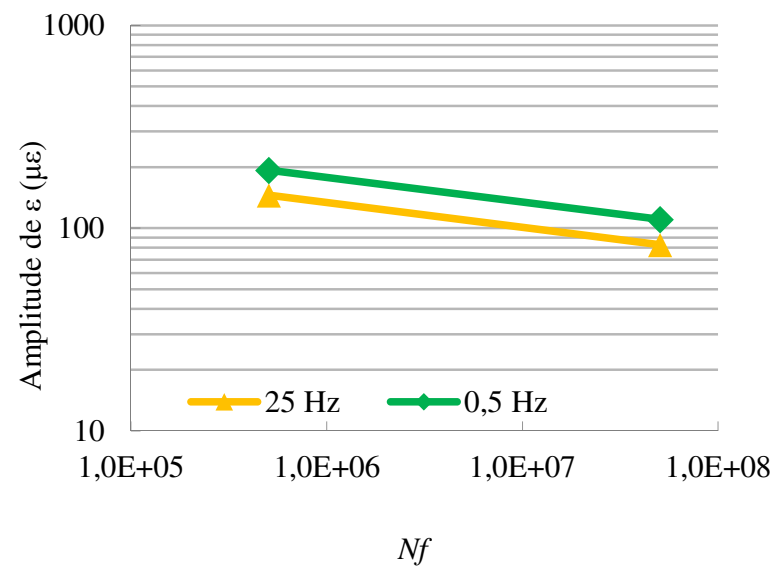

(b) Variação de frequência $(25$ e $0,5 \mathrm{~Hz})$ e temperatura fixa $(10 \circ \mathrm{C})$

Figura 5. Curvas de Wöhler de M4 variando temperaturas e frequências

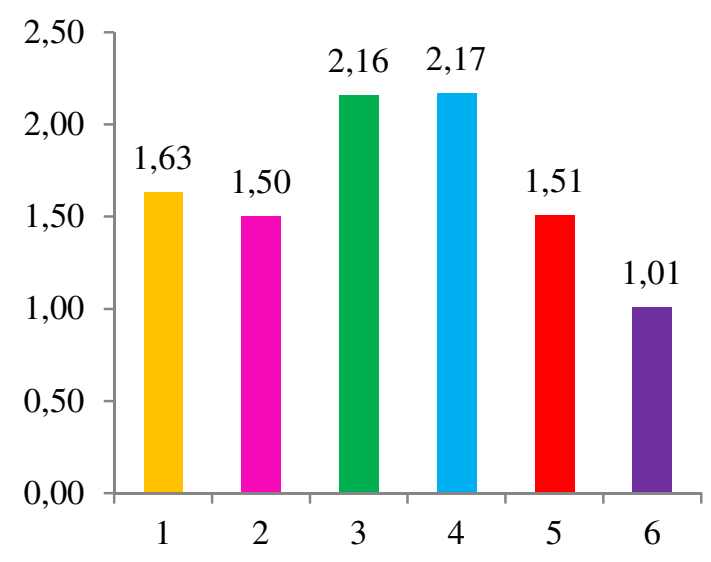

(a) FFM $(100-200 \mu \varepsilon)$

Figura 6. FFM e classificação de resistência à fadiga das misturas avaliadas

\section{CONCLUSÕES}

A partir de um banco de dados de 54 misturas asfálticas foram calculados todos os critérios FAD-CI. Escolheram-se 6 misturas (M1, M2, M3, M4, M5 e M6), de tal forma que a primeira mistura não atendesse qualquer critério e as demais atendessem crescentemente aos critérios da citada metodologia ( $\eta$ FAD, FR, RAF e EEFL). Das 54 misturas, apenas duas (M5 e M6) enquadravam-se no intervalo desejado de EEFL, sendo que estas atendiam apenas ao mesmo. 
Em seguida, simularam-se as vidas de fadiga das 6 misturas com o intuito de comparar o ranqueamento feito a partir da metodologia FAD-CI, e também dos resultados das simulações. Observou-se ainda o FFM e as classes de resistência à fadiga das misturas. As seguintes conclusões foram possíveis:

- A mistura que não atende qualquer critério da metodologia FAD-CI (M1) e a que atende apenas a porosidade FAD (M2) apresentam menores resistências à fadiga. Portanto, quando se observam as curvas de Wöhler, percebe-se que a metodologia tem potencial de ajudar na etapa de seleção granulométrica, pois misturas que se enquadraram nos intervalos ótimos dos parâmetros tendem a apresentar maior resistência à fadiga.

- Quando se analisam as misturas que atendem mais critérios da metodologia FAD-CI (M3 e M4), percebe-se que ambas apresentaram desempenho superior na maioria das análises, sobretudo a $10^{\circ} \mathrm{C}$. Porém, devem-se conduzir pesquisas considerando misturas dosadas com os mesmos CAP e agregados (mesma formação mineralógica e TMN). Dessa forma, acredita-se que será possível identificar com mais clareza a influência dos parâmetros da metodologia FAD-CI na suscetibilidade à fadiga, superando algumas das limitações do presente artigo.

- M5 e M6, misturas que atendem apenas o parâmetro EEFL, apresentaram um desempenho superior nas análises com maior valor de temperatura $\left(40^{\circ} \mathrm{C}\right)$. Logo, este parâmetro específico demonstrou ser relevante e importante de ser observado no projeto de misturas. Acredita-se que misturas que atendem o parâmetro EEFL podem apresentar maior resistência à fadiga quando aplicadas em locais com temperaturas elevadas. M5 e M6 possuem cal como fíler e seus TMNs são os maiores dentre as misturas avaliadas, sendo que estas características afetaram o EEFL e, por conseguinte, influenciaram os seus comportamentos mecânicos.

- Pode-se concluir, ao observar os índices FFM e classes das misturas, que M3 e M4 atenderam mais parâmetros FAD-CI (sobretudo FR e RAF) e mostraram-se, respectivamente, como as mais resistentes à fadiga. Estas misturas também foram ranqueadas como as mais resistentes ao observar as curvas de Wöhler. Independente do parâmetro de fadiga escolhido (curva de Wöhler ou FFM e classes das misturas), as misturas que mais atenderam parâmetros FAD-CI apresentaram-se como as mais resistentes à fadiga. Concluise então que atender ao maior número possível de parâmetros FAD-CI pode incrementar a performance das misturas com vistas a diminuir a susceptibilidade à fadiga.

\section{REFERÊNCIAS}

AASHTO T 342 (2011) Standard Method of Test for Determining Dynamic Modulus of Hot Mix Asphalt (HMA). Washington, D.C.USA.

AASHTO TP 107 (2018) Method of Test For Determining the Damage Characteristic Curve of Asphalt Mixtures From Direct Tension Cyclic Fatigue Tests. American Association of State Highway and Transportation Officials. Washington, D.C.-USA.

Almeida, F. J. P. (2019) Modelagem Computacional de Misturas Asfálticas com Utilização do Método dos Elementos Finitos e da Teoria do Dano Contínuo. Tese (doutorado). Programa de Pós-Graduação em Engenharia de Transportes, Universidade Federal do Ceará (UFC). Fortaleza-CE. Disponível em: <http://www.repositorio.ufc.br/handle/riufc/40405> (acesso em 18/08/2021).

ASTM E1823 (2013) Standard Terminology Relating to Fatigue and Fracture Testing, ASTM International. Pennsylvania-USA.

Babadopulos, L. F. A. L. (2014) A Contribution to Couple Aging to Hot Mix Asphalt (HMA) Mechanical Characterization Under Load-Induced Damage. Dissertação (mestrado). Programa de Pós-Graduação em Engenharia de Transportes, Universidade Federal do Ceará (UFC). Fortaleza-CE. Disponível em: <http://www.repositorio.ufc.br/handle/riufc/11164> (acesso em 18/08/2021). 
Babadopulos, L. F. A. L; J. B. Soares e V. T. F. Castelo Branco (2015) Interpreting Fatigue Tests in Hot Mix Asphalt (HMA) Using Concepts from Viscoelasticity and Damage Mechanics. Revista Transportes, v. 23, n. 2, p. 85-94. D0I: 10.14295/transportes.v23i2.898

Chun, S. e K. Kim (2016) Effectiveness of Dominant Aggregate Size Range - Interstitial Component Criteria For Consistently Enhanced Cracking Performance of Asphalt Mixtures in the Field. Canadian Journal of Civil Engineering, v. 43, p. 523-531. DOI: 10.1139/cjce-2015-0517

CNT (2018) Pesquisa CNT de Rodovias 2018: Relatório Gerencial. CNT, SEST, SENAT. Distrito Federal - Brasília.

DNIT 031 (2016) Pavimentos Flexíveis - Concreto Asfáltico. Departamento Nacional de Infraestrutura de Transportes, Instituto se Pesquisas Rodoviárias. Rio de Janeiro-RJ.

DNIT 183 (2018) Pavimentação Asfáltica - Ensaio de Fadiga por Compressão Diametral à Tensão Controlada. Departamento Nacional de Infraestrutura de Transportes, Instituto De Pesquisas Rodoviárias. Rio de Janeiro-RJ.

Fengler, R. Z. (2018) Caracterização de Ligantes e Misturas Asfálticas Modificados com a Adição de Trinidad Lake Asphalt. Dissertação (mestrado). Programa de Pós-Graduação em Engenharia Civil, Universidade Federal do Rio de Janeiro (UFRJ). Rio de Janeiro-RJ. Disponível em: <http://hdl.handle.net/11422/13418> (acesso em 18/08/2021).

Ferreira, J. L. S. (2015) Melhoramento da Resistência à Deformação Permanente de Misturas Asfálticas pela Escolha Granulométrica a partir da Metodologia da Faixa de Agregados Dominantes. Monografia (iniciação cientifica). Projeto de Graduação Universidade Federal do Ceará, Fortaleza-CE.

Greene, J.; S. Chum e B. Choubane (2014) Enhanced Gradation Guidelines to Improve Asphalt Mixture Performance. Transportation Research Record, v. 2456, p. 3-10. https://doi.org/10.3141/2456-01

Guarin, A. (2009) Interstitial Component Characterization to Evaluate Asphalt Mixture Performance. Tese (doutorado). Universidade da Florida. Gainesville-USA. Disponível em: <https://ufdc.ufl.edu/UFE0024951/00001> (acesso em 31/08/2021).

Hou, T.; B. S. Underwood e Y. R. Kim (2010) Fatigue Performance Prediction of North Carolina Mixtures Using the Simplified Viscoelastic Continuum Damage Model. Asphalt Paving Technology: Association of Asphalt Paving Technologists-Proceedings of the Technical Sessions, v. 79, p. 35-73.

Kim, S. (2006) Identification and Assessment of the Dominant Aggregate Size Range of Asphalt Mixture. Tese (doutorado). Universidade da Florida. Gainesville-USA. Disponível em: <https://ufdc.ufl.edu/UFE0013464/00001> (acesso em 31/08/2021).

Kim, Y. R.; M. N. Guddati; B. S. Underwood; T. Y. Yun; V. Subramanian; S. Savadatti e S. Thirunavukkarasu (2009) Development of a Multiaxial Viscoelastoplastic Continuum Damage Model for Asphalt Mixtures. Publication FHWA-HRT-08-073, U.S. Department of Transportation, Federal Highway Administration.

Monismith, C. L. (1998) Asphalt Concrete: An Extraordinary Material for Engineering Applications. Thirtieth Henry M. Shaw Lecture in Civil Engineering. Department of Civil Engineering, College of Engineering North Carolina State University Raleigh. North Carolina State University. North Carolina, USA.

Nascimento, L. A. H. (2015) Implementation and Validation of the Viscoelastic Continuum Damage Theory for Asphalt Mixture and Pavement Analysis in Brazil. Tese (doutorado). Universidade Estadual da Carolina do Norte. Raleigh-EUA. Disponível em: < https://repository.lib.ncsu.edu/handle/1840.16/10651> (acesso em 31/08/2021).

Nascimento, L. A. H. (2016) Apresentação Realizada no Departamento de Engenharia de Transportes na Universidade Federal do Ceará. Data: 21 de dezembro de 2016, Fortaleza-CE.

Nascimento, L. A. H. (2018) Apresentação Realizada no CT-Asfalto N/NE na Universidade Federal do Ceará. Data: 12 de dezembro de 2018, Fortaleza-CE.

Nukunya, B.; R. Roque; M. Tia e B. Birgisson (2001) Evaluation of VMA and Other Volumetric Properties as Criteria for the Design and Acceptance of Superpave Mixtures. Journal of the Association of Asphalt Paving Technologists, v. 70 , p. 38 - 69.

Portela Neto, M. G. (2018) Avaliação da Porosidade Marginal da Faixa de Agregados Dominantes em Misturas Asfálticas. Dissertação (mestrado). Programa de Pós-Graduação em Engenharia de Transportes, Universidade Federal do Ceará (UFC). Fortaleza-CE. Disponível em: <http://www.repositorio.ufc.br/handle/riufc/49901> (acesso em 18/08/2021).

Santiago, L. S.; L. F. A. L. Babadopulos e J. B. Soares (2019) Desenvolvimento de Função de Transferência para Previsão de Área Trincada em Pavimentos Asfálticos por Meio da Simulação do Dano por Fadiga Utilizando Modelo S-VECD e Análises Elásticas. Revista Transportes v. 28, n. 3, p. 121-136. https://doi.org/10.14295/transportes.v28i3.1900

Santiago, L. S.; S. A. T. Silva e J. B. Soares (2018) Determinação do Dano em Pavimentos Asfálticos por Meio da Combinação do Modelo S-VECD com Análises Elásticas. Revista Transportes v. 21, n. 2, p. 31 - 43. https://doi.org/10.14295/transportes.v28i3.1900

Sengoz, B. e A. Topal (2007) Minimum Voids in Mineral Aggregate in Hot-Mix Asphalt Based on Asphalt Film Thickness. Journal of Building and Environment v. 42, p. 3629 - 3635. https://doi.org/10.1016/j.buildenv.2006.10.005

Underwood, B. S.; H. E. T. Hou e Y. R. Kim (2009) Application of Simplified VECD Modeling to the Fatigue Prediction of Asphalt Concrete Mixtures. 7 th International RILEM Symposium on Advanced Testing and Characterization of Bituminous Materials. Rhodes-Greece.

WHO (2018) Global Status Report on Road Safety - World Health Organization. CC BYNC-SA 3.0 IGO. Genebra-Suíça.

Zhou, F.; S. Hu e T. Scullion (2006) Integrated Asphalt (Overlay) Mixture Design Balancing Rutting and Cracking Requirements. Texas Department of Transportation and Federal Highway Administration Report 0-5123-1. Disponível em: <https://static.tti.tamu.edu/tti.tamu.edu/documents/0-5123-1.pdf> (acesso em 18/08/2021). 